\title{
The widths of quarkonia in quark gluon plasma
}

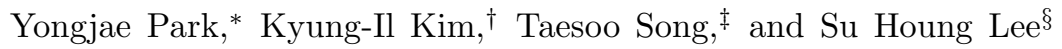 \\ Institute of Physics and Applied Physics, Yonsei University, Seoul 120-749, Korea
}

Cheuk-Yin Wong

Physics Division, Oak Ridge National Laboratory, Oak Rige, TN 37830 and

Department of Physics, University of Tennessee, Knoxville, TN 37996

\begin{abstract}
Recent lattice calculations showed that heavy quarkonia will survive beyond the phase transition temperature, and will dissolve at different temperatures depending on the type of the quarkonium. In this work, we calculate the thermal width of a quarkonium at finite temperature before it dissolves into open heavy quarks. The input of the calculation are the parton quarkonium dissociation cross section to NLO in QCD, the quarkonium wave function in a temperature-dependent potential from lattice QCD, and a thermal distribution of partons with thermal masses. We find that for the $J / \psi$, the total thermal width above $1.4 T_{c}$ becomes larger than 100 to $250 \mathrm{MeV}$, depending on the effective thermal masses of the quark and gluon, which we take between 400 to $600 \mathrm{MeV}$. Such a width corresponds to an effective dissociation cross section by gluons between 1.5 to $3.5 \mathrm{mb}$ and by quarks 1 to $2 \mathrm{mb}$ at $1.4 T_{c}$. However, at similar temperatures, we find a much smaller thermal width and effective cross section for the $\Upsilon$.
\end{abstract}

PACS numbers: $13.20 . \mathrm{He}, 14.20 . \mathrm{Lq}$

Keywords:

\section{INTRODUCTION}

Recently, a number of important progress have been made in the physics of $J / \psi$ suppression as a signature of quark gluon plasma that inevitably leads us to augment the original work by Matsui and Satz [1] with a more detailed study of heavy quark system at finite temperatures, before confronting the recent RHIC data[2], and in predicting results for LHC. Among these theoretical developments are the phenomenologically successful statistical model for $J / \psi$ production 3 , , 4, 5], based on a coalescence assumption near $T_{c}[5]$, the recombination of charm pairs into $J / \psi[6]$, and the recent lattice calculations, showing strong evidence that the heavy quarkonium will persist above $T_{c}[7,8,9,9,10,11]$. While these results seem at odd with each other, it only suggests that one still needs a more detailed understanding of the properties of heavy quark system in the quark gluon plasma, especially between the phase transition and the dissolving temperatures, before a consistent picture of quarkonium suppression in heavy ion collision can be achieved.

In this respect, an important quantity to investigate is the effective thermal width, and/or the effective dissociation cross section of a heavy quarkonium in the quark gluon plasma. Except for its existence, the present lattice results are far from making quantitative statements on the magnitude of thermal width for charmonium states above $T_{c}$. Hence, in this work, we will use the perturbative QCD approach to calculate the thermal width. So far, such calculations have been limited to dissociation processes by gluons to the lowest order
(LO) 12, 13, 14, 15, 16, 17], because the elementary $J / \psi$ parton dissociation cross section was available only to that order 18], and to part of the next-to-leading-order (NLO) in the quasi free approximation[19]. Recently, two of us have performed the dissociation cross section calculation to NLO in QCD 20]. Here, we will implement the NLO formula, to calculate the corresponding thermal width of charmonium [21], and then perform a similar calculation for the bottonium case.

The NLO calculation of $J / \psi$-parton dissociation calculations involves collinear divergence. When applying this elementary cross section to dissociation by hadrons, the collinear divergence is cured by mass factorization, which renormalizes the divergent part of the cross section into the parton distribution function of the hadron. Such complications disappear at finite temperatures, as the thermal masses of the partons automatically renders the divergence finite. The magnitude of the thermal mass as a function of temperature has been obtained previously by examining the equation of state [22]. In the region of $T_{c}$ to $2 T_{c}$, they are of the order of $300-400 \mathrm{MeV}$ for quarks and 400-600 MeV for gluons. In this work, instead of following the detailed temperature dependence, we shall study results with a thermal mass of 400 and $600 \mathrm{MeV}$ for both the quarks and gluons. As we will see, with an effective thermal mass of $400 \mathrm{MeV}$, we find that the effective thermal dissociation cross section above 1.4 $T_{c}$ is larger than $250 \mathrm{MeV}$. The NLO calculation is proportional to the derivative of the momentum space wave function. We will use the Coulomb wave function, whose size have been fitted to reproduce the result obtained by 


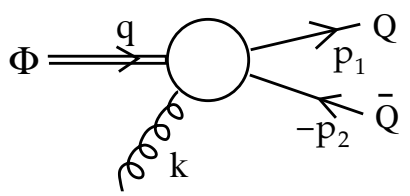

FIG. 1: LO diagram

Wong [16], using a potential extracted from lattice gauge thermodynamical quantities.

In Section II, we will recapitulate the LO result. In Section III, we will discuss the NLO for $J / \psi$. The LO and NLO results for bottonium are given in Section IV.

\section{LO RESULT}

The LO invariant matrix element for the $J / \psi$ dissociation cross section by gluon first obtained by Peskin 18] and rederived by one of us [23] using the Bethe-Salpeter equation is given as,

$$
\begin{aligned}
\mathcal{M}^{\mu \nu}= & -g \sqrt{\frac{M_{\Phi}}{N_{c}}}\left\{\mathbf{k} \cdot \frac{\partial \psi(\mathbf{p})}{\partial \mathbf{p}} \delta^{\nu 0}+k_{0} \frac{\partial \psi(\mathbf{p})}{\partial p^{j}} \delta^{\nu j}\right\} \delta^{\mu i} \\
& \times \bar{u}\left(p_{1}\right) \frac{1+\gamma_{0}}{2} \gamma^{i} \frac{1-\gamma_{0}}{2} T^{a} v\left(p_{2}\right)
\end{aligned}
$$

Here, $\mu, \nu$ represents the polarization index of the $J / \psi$ and gluon respectively, and $k, p_{1}, p_{2}$ are the four momentum of the gluon, $c, \bar{c}$. The quantity $\mathbf{p}$ is the relative three momentum between $c$ and $\bar{c}$, and $\psi(\mathbf{p})$ is the charmonium wave function. $M_{\Phi}$ is the mass of a quarkonium, and $N_{c}$ is the number of color. Current conservation is easily shown to be satisfied, $k_{\nu} M^{\mu \nu}=0$. The energy conservation in the non-relativistic limit implies,

$$
k_{0}+m_{\Phi}=2 m_{c}+\frac{\left|\overrightarrow{p_{1}}\right|^{2}+\left|\overrightarrow{p_{2}}\right|^{2}}{2 m_{c}},
$$

from which, the counting scheme for both the $\mathrm{LO}$ [18] and the NLO [20] are given as follows,

$$
\begin{aligned}
\left|\overrightarrow{p_{1}}\right| \sim\left|\overrightarrow{p_{2}}\right| \sim|\vec{p}| \sim O\left(m g^{2}\right) \\
k^{0} \sim|\vec{k}| \sim O\left(m g^{4}\right) .
\end{aligned}
$$

The effective thermal width and cross section are obtained by folding the matrix element with the thermal parton distribution, $n\left(k_{0}\right)$

$$
\begin{aligned}
\Gamma^{e f f} & =d_{p} \int \frac{d^{3} k}{(2 \pi)^{3}} n\left(k_{0}\right) v_{r e l} \sigma\left(k_{0}\right), \\
\sigma^{e f f} & =\int \frac{d^{3} k}{(2 \pi)^{3}} n\left(k_{0}\right) \sigma\left(k_{0}\right) / \int \frac{d^{3} k}{(2 \pi)^{3}} n\left(k_{0}\right),
\end{aligned}
$$

where $d_{p}$ is the parton degeneracy, which is taken to be 16 for the gluon in the LO calculation. Here, we will

\begin{tabular}{|c|c|c|c|c|c|c|}
\hline$T / T_{c}$ & 1.13 & 1.18 & 1.25 & 1.40 & 1.60 & 1.65 \\
\hline$\epsilon_{0}(\mathrm{MeV})$ & 36.4 & 20.9 & 10.1 & 3.4 & 0.14 & 0.004 \\
\hline$\sqrt{<r^{2}>}(\mathrm{fm})$ & 0.97 & 1.19 & 1.54 & 2.30 & 4.54 & 5.17 \\
\hline$a_{0}(\mathrm{fm})$ & 0.56 & 0.69 & 0.89 & 1.33 & 2.62 & 2.99 \\
\hline
\end{tabular}

TABLE I: The binding energy, the rms radius, and its corresponding Bhor radius of $J / \psi$ at finite temperature.

perform the calculation in the rest frame of $J / \psi$, so the relative velocity of $J / \psi$ and initial parton is $v_{r e l}=\left|\vec{k} / k_{0}\right|$. The cross section is given as,

$$
\begin{gathered}
\sigma=\int \frac{1}{128 \pi^{2} m_{\Phi}|\vec{k}|} \sqrt{\frac{k_{0}-\epsilon_{0}}{m_{c}}} \overline{\mathcal{M}}^{2} d \Omega \\
\overline{|\mathcal{M}|}^{2}=\frac{2 g^{2} m_{c}^{2} m_{\Phi}\left(2 k_{0}^{2}+m_{k_{1}}^{2}\right)}{3 N_{c}}\left|\frac{\partial \psi(\mathbf{p})}{\partial \mathbf{p}}\right|^{2}
\end{gathered}
$$

where $m_{k_{1}}$ is the thermal mass of a gluon. As can be seen from Eq.(5), the cross section is proportional to the absolute square of the derivative of the momentum space wave function, which comes from the dipole nature of the interaction between the gluon and the quark antiquark pair with opposite charges. In the calculation of the effective thermal width or the effective cross section in Eq.(4), the cross section is integrated over the incoming energy, which effectively integrates over the absolute square of the derivative of the momentum space wave function. As a consequence, the results are sensitive to the size of the wave function only and not so much on its detailed functional form. Therefore, we will use a Coulomb wave function, whose Bohr radius is fitted to reproduce the rms radius obtained by one of us [16] by solving the bound states in a temperature-dependent potential extracted from lattice gauge thermodynamical quantities. For the binding energy, we use the values obtained in ref. [16]. Table【summarizes the $J / \psi$ binding energy, its rms radius, and its corresponding Bhor radius at finite temperature. The coupling constant $g$ is set such that $\alpha_{s}$ is 0.5. As can be seen in Fig. 2, $\sigma\left(k_{0}\right)$ in the LO is dominant around the $J / \psi$ binding energy [16], which decreases as temperature increases. On the other hand, $n\left(k_{0}\right)$ favors higher $k_{0}$ as temperature increases. Hence the overlap in Eq.(4) decreases at higher temperature, more so because the overlap integral starts from the thermal mass of the gluon. As can be seen in Fig. 3, choosing the effective thermal mass of the gluons to be 400 and $600 \mathrm{MeV}$, which is much larger than the binding energy, we find that the effective thermal width decreases as the temperature increases and becomes very small. With lower bound of the thermal mass $(400 \mathrm{MeV})$ the width at LO is smaller than $3 \mathrm{MeV}$ at $1.13 T_{c}$ and less than $1 \mathrm{MeV}$ at $1.4 T_{c}$. At the NLO, there are other thermal gluon and quark induced interaction reactions representatively shown in Fig. 4. 
The width due to these contributions will be calculated in the next section.

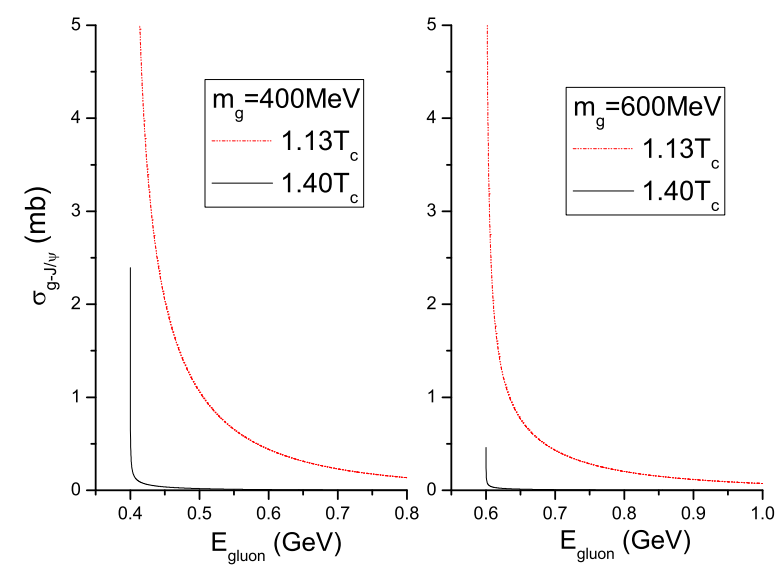

FIG. 2: (Color online) $\sigma\left(E_{\text {gluon }}\right)$ of $J / \psi$ at LO.

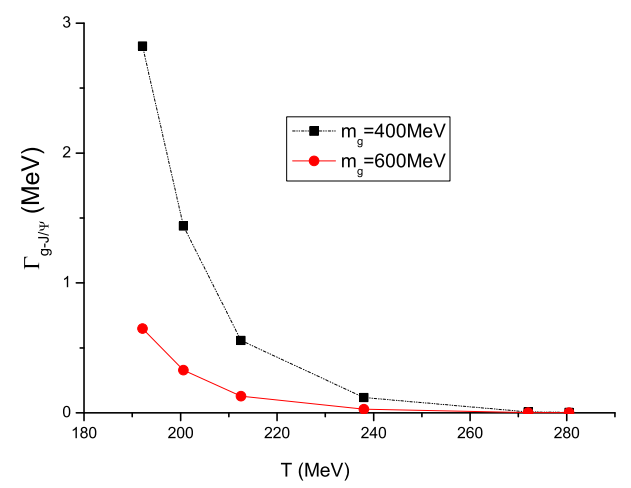

FIG. 3: (Color online) Effective thermal width of $J / \psi$ at LO. The dots and circles show the results at temperatures given in Table $\Pi$ with $T_{c}=170 \mathrm{MeV}$.

\section{NLO RESULT}

The $J / \psi$ dissociation cross section by partons in QCD at the NLO was performed by two of us 20]. The dissociation cross section can be divided into two parts; the dissociation by quarks and that by gluons (Fig. 4).

In both cases, the cross sections are given as,

$$
\sigma(u)=\frac{1}{4 \sqrt{\left(q \cdot k_{1}\right)^{2}-m_{k_{1}}^{2} m_{\Phi}^{2}}} \int d \sigma_{3} \mid \overline{\mathcal{M}}^{2}
$$

where $u^{2}=\left(q+k_{1}\right)^{2}, m_{k_{1}}$ is the thermal mass of a parton, and $d \sigma_{3}$ is the 3-body phase space,

$$
\begin{aligned}
d \sigma_{3}= & \frac{d^{3} k_{2}}{(2 \pi)^{3} 2 k_{20}} \frac{d^{3} p_{1}}{(2 \pi)^{3} 2 p_{10}} \frac{d^{3} p_{2}}{(2 \pi)^{3} 2 p_{20}} \\
& \times(2 \pi)^{4} \delta^{(4)}\left(q+k_{1}-k_{2}-p_{1}-p_{2}\right)
\end{aligned}
$$

Here, $q, k_{1}, k_{2}, p_{1}$ and $p_{2}$ are respectively the momentum of $J / \psi$, incoming parton, outgoing parton, charm quark, and anti-charm quark.

In the $J / \psi$ rest frame, the cross section can be written as,

$$
\begin{aligned}
\sigma= & \frac{1}{4 \sqrt{\left(q \cdot k_{1}\right)^{2}-m_{\Phi}^{2} m_{k_{1}}^{2}}} \\
& \times \int_{\alpha}^{\beta} d w^{2} \int_{\alpha^{\prime}}^{\beta^{\prime}} d p_{\Delta}^{2} \frac{\sqrt{1-4 m_{c}^{2} / w^{2}}}{16^{2} \pi^{3} m_{\Phi}\left|\overrightarrow{k_{1}}\right|} \mid \overline{\mathcal{M}}^{2},
\end{aligned}
$$

where $p_{\Delta}^{2}=\left(k_{1}-k_{2}\right)^{2}, w^{2}=\left(q+p_{\Delta}\right)^{2}$. The integration range is

$$
\begin{aligned}
\alpha & =\left(m_{p_{2}}+m_{p_{1}}\right)^{2}=4 m_{c}^{2}, \\
\beta & =\left(u-m_{k_{1}}\right)^{2}, \\
\alpha^{\prime} & =-b-\sqrt{b^{2}-a c}, \\
\beta^{\prime} & =-b+\sqrt{b^{2}-a c},
\end{aligned}
$$

where

$$
\begin{aligned}
b= & \frac{1}{2 u^{2}}\left\{u^{2}-\left(m_{\Phi}+m_{k_{1}}\right)^{2}\right\}\left\{u^{2}-\left(m_{\Phi}-m_{k_{1}}\right)^{2}\right\} \\
& -\frac{1}{2 u^{2}}\left\{u^{2}-\left(m_{\Phi}^{2}-m_{k_{1}}^{2}\right)\right\}\left\{w^{2}-m_{\Phi}^{2}\right\}, \\
b^{2}-a c= & \frac{1}{4 u^{4}}\left\{\left(u^{2}-m_{\Phi}^{2}+m_{k_{1}}^{2}\right)^{2}-4 u^{2} m_{k_{1}}^{2}\right\} \\
& \times\left\{w^{2}-\left(u+m_{k_{1}}\right)^{2}\right\}\left\{w^{2}-\left(u-m_{k_{1}}\right)^{2}\right\} .
\end{aligned}
$$

The variables appearing in $\sigma$ can be expressed in terms of $w^{2}, p_{\Delta}$ and $u^{2}$ as follows,

$$
\begin{aligned}
q \cdot k_{1} & =\left(u^{2}-m_{\Phi}^{2}-m_{k_{1}}^{2}\right) / 2, \\
\left|\overrightarrow{k_{1}}\right| & =\sqrt{\left\{\left(u^{2}-m_{k_{1}}^{2}+m_{\Phi}^{2}\right) /\left(2 m_{\Phi}\right)\right\}^{2}-u^{2}} \\
k_{1} \cdot k_{2} & =\left(-p_{\Delta}^{2}+2 m_{k_{1}}^{2}\right) / 2 \\
k_{10} & =\sqrt{\left|\overrightarrow{k_{1}}\right|^{2}+m_{k_{1}}^{2}} \\
k_{20} & =k_{10}-\left(w^{2}-p_{\Delta}^{2}-m_{\Phi}^{2}\right) /\left(2 m_{\Phi}\right) \\
|\vec{p}| & =\sqrt{m_{c}\left(k_{10}-k_{20}+m_{\Phi}-2 m_{c}\right)} .
\end{aligned}
$$
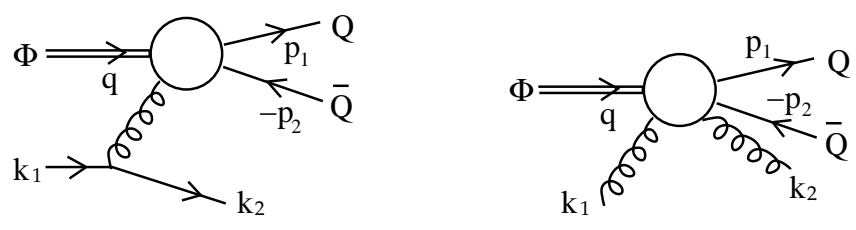

FIG. 4: NLO diagrams induced by (a)quarks, (b)gluons. 


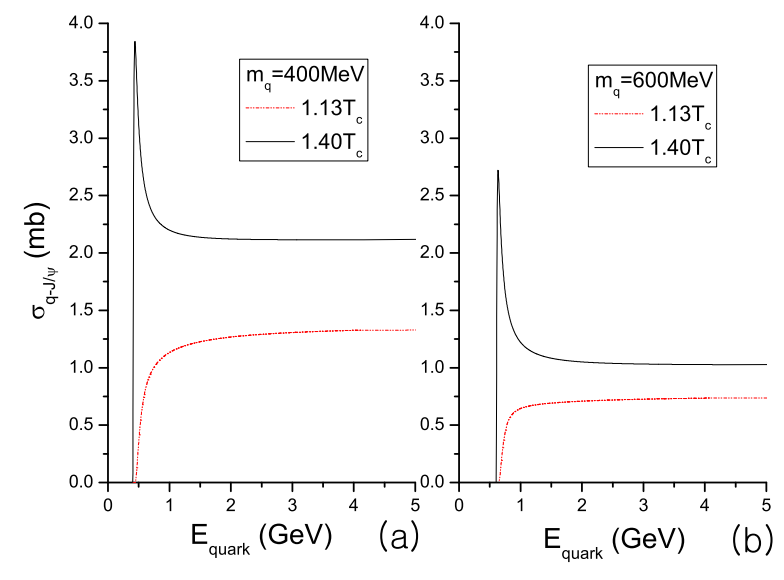

FIG. 5: (Color online) $\sigma\left(E_{\text {quark }}\right)$ of $q-J / \psi$ at NLO.

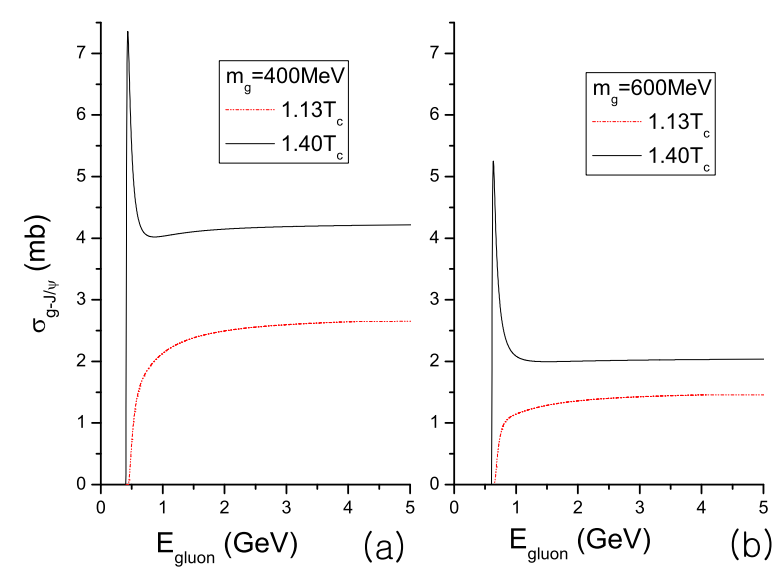

FIG. 6: (Color online) $\sigma\left(E_{\text {gluon }}\right)$ of $g-J / \psi$ at NLO.

We first consider the NLO effective thermal width induced by quark. Here, the quark degeneracy is 36 assuming 3 flavors. The invariant matrix element is given as 20],

$$
\overline{\mid \mathcal{M}}^{2}=\frac{4}{3} g^{4} m_{c}^{2} m_{\Phi}\left|\frac{\partial \psi(\mathbf{p})}{\partial \mathbf{p}}\right|^{2}\left(-\frac{1}{2}+\frac{k_{10}^{2}+k_{20}^{2}}{2 k_{1} \cdot k_{2}}\right) .
$$

Next, the gluon induced NLO calculation has the same degeneracy as the LO case, and the invariant matrix element is given as follows 20],

$$
\begin{aligned}
& \overline{|\mathcal{M}|}^{2}=\frac{4}{3} g^{4} m_{c}^{2} m_{\Phi}\left|\frac{\partial \psi(\mathbf{p})}{\partial \mathbf{p}}\right|^{2}\left\{-4+\frac{k_{1} \cdot k_{2}}{k_{10} k_{20}}\right. \\
& +\frac{2 k_{10}}{k_{20}}+\frac{2 k_{20}}{k_{10}}-\frac{k_{20}^{2}}{k_{10}^{2}}-\frac{k_{10}^{2}}{k_{20}^{2}}+\frac{2}{k_{1} \cdot k_{2}} \\
& \left.\times\left(\frac{\left(k_{10}^{2}+k_{20}^{2}\right)^{2}}{k_{10} k_{20}}-2 k_{10}^{2}-2 k_{20}^{2}+k_{10} k_{20}\right)\right\} .
\end{aligned}
$$

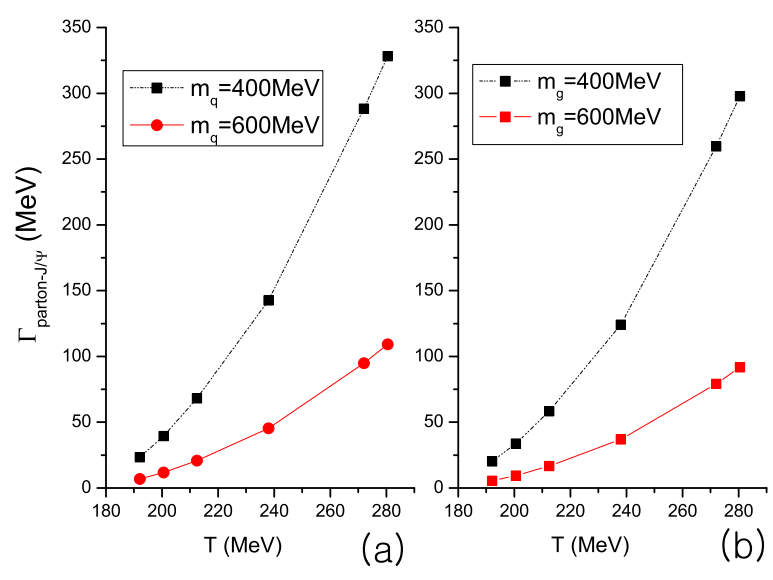

FIG. 7: (Color online) Effective thermal width of $J / \psi$ at NLO induced (a) by quarks and (b) by gluons

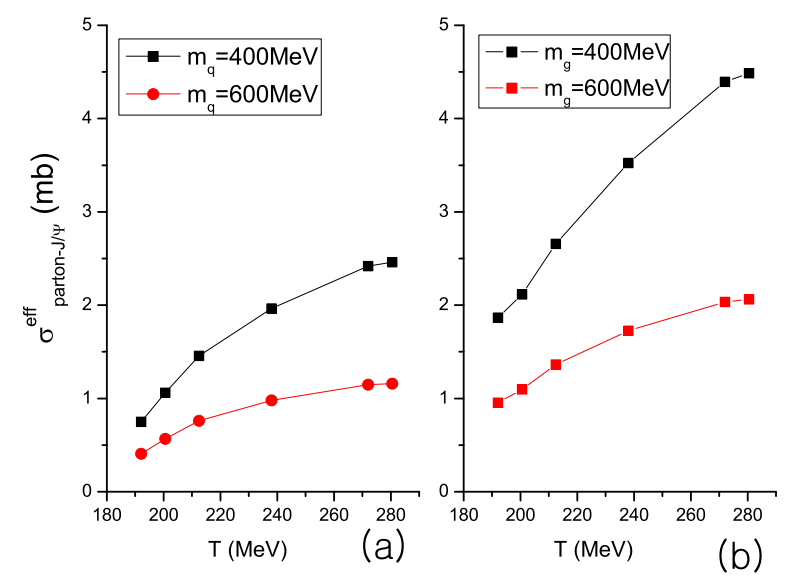

FIG. 8: (Color online) Effective cross-section of $J / \psi$ induced (a) by quarks (NLO) and (b) by gluons (LO+NLO)

In the hadronic phase, the $1 /\left(k_{1} \cdot k_{2}\right)$ term, and the $1 / k_{20}^{2}$ term give rise to collinear divergence, and soft divergence respectively. However, in QGP phase, the thermal mass of a parton $m_{k_{1}}$ in Eq.(10) plays the role of a cutoff. In contrast to the LO calculation, as can be seen from Fig. 5 for quark and from Fig. 6 for gluon, $\sigma\left(k_{0}\right)$ at the NLO does not vanish at large $k_{0}$. This is so because irrespective of how large the energies of the incoming quark or the gluon are, they can always radiate small energy gluon in the order of the binding energy to effectively dissociate $J / \psi$ via the LO process. Hence, in Eq. (4), $\sigma$ has non trivial overlap with the maximum of $n\left(k_{0}\right)$ that increase with temperature, leading to the result shown in Fig. 7(a) and in Fig. 7(b) for quark and gluon induced NLO width respectively. One thing to note from Fig. [5 is that the elementary cross 
section has a peak near threshold at $1.4 T_{c}$. Such peak structure only appears when the binding energy becomes very small and the corresponding momentum space wave function becomes highly peaked near zero momentum. When the incoming energy is small, these highly peaked region gives important contributions to the two dimensional phase space integral in Eq. (8). But when the incoming energy becomes large, the phase space for the peaked region becomes smaller and so does the total cross section. Such singular behavior as a function of the incoming energy disappears when the binding energy becomes larger.

Here we take the thermal mass from $400 \mathrm{MeV}$ to 600 $\mathrm{MeV}$ [22] within the temperature region of a few $T_{c}$. With those masses we obtained large thermal widths. Even with an upper limit thermal mass of $600 \mathrm{MeV}$, the width exceeds $100 \mathrm{MeV}$ above $1.4 T_{c}$, where we have taken $T_{c}=170 \mathrm{MeV}$. For example, if the thermal mass of partons is $600 \mathrm{MeV}$, and the produced $J / \psi$ remains at 1.4 $T_{c}$ for $2 \mathrm{fm} / \mathrm{c}$, its survival rate will be less than $40 \%$. As can be seen from Fig. 8(a) and Fig. 8(b), with a 600 $\mathrm{MeV}$ thermal mass at 1.4 $T_{c}$, the effective dissociation cross section by a quark is about $1.0 \mathrm{mb}$ and that by a gluon $1.5 \mathrm{mb}$. Hence, even though the $J / \psi$ might start forming at 1.6 $T_{c}$, its effective width is very large and will not accumulate until the system cools down further.

\section{$\Upsilon$ DISSOCIATION}

Here, we present the result for the $\Upsilon$ case. The $\Upsilon$ wave function is less sensitive to changes in the temperature [16]. In the LO calculation, while the trends in the temperature dependence of $\sigma$ is similar to that of the $J / \psi$, its variation in magnitudes is much smaller. Moreover, as can be seen in Table \the binding energy remains large. As a consequence, the overlap of $\sigma$ and the thermal distribution remains large even at large temperatures, and the effective thermal width slowly decreases

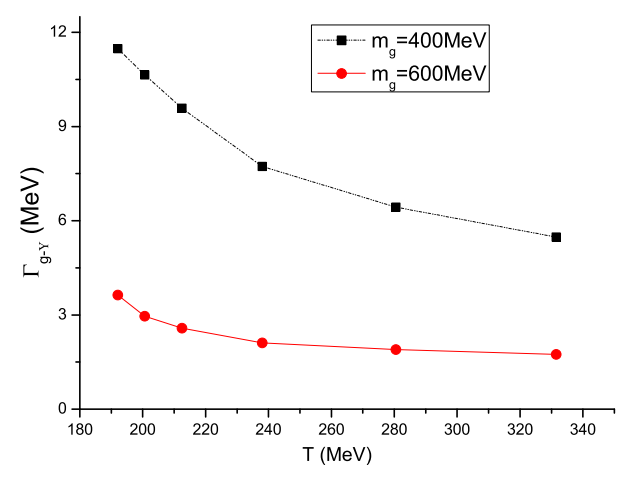

FIG. 9: (Color online) Effective thermal width of $\Upsilon$ at LO

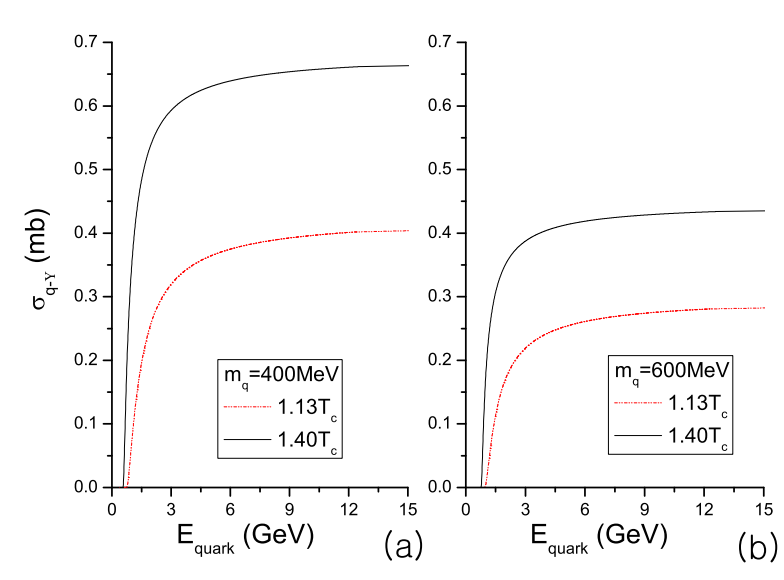

FIG. 10: (Color online) $\sigma\left(E_{\text {quark }}\right)$ of $q-\Upsilon$ at NLO.

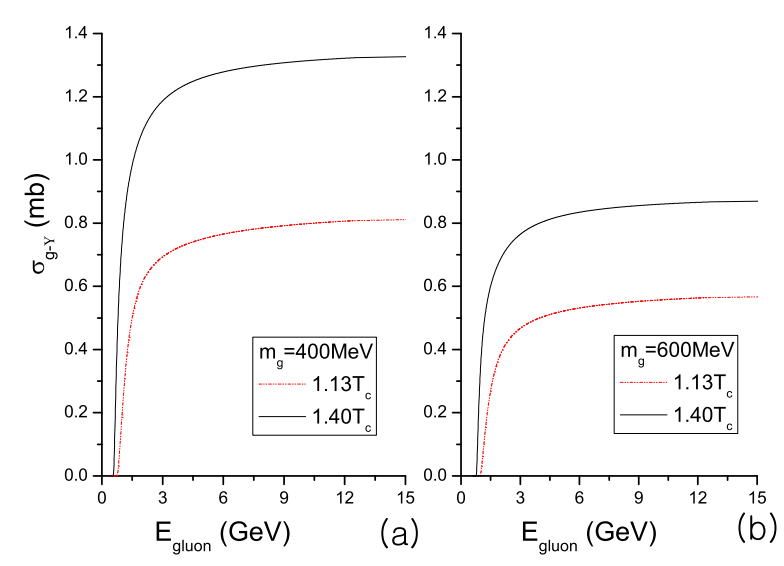

FIG. 11: (Color online) $\sigma\left(E_{\text {gluon }}\right)$ of $g-\Upsilon$ at NLO.

with temperature as can be seen in Fig. 9,

In the NLO calculation, as shown in Fig. 10, and Fig. 11. the form of the cross section is similar to that of $J / \psi$. However, because the binding energy of $\Upsilon$ is still large at high temperature, there is no peak structure near threshold. Moreover, because $\Upsilon$ is more tightly bound and has a smaller dipole size than $J / \psi$, its corresponding $\sigma$ is also smaller. Therefore, as can be seen from Fig. 12(a) for the quark induced and Fig. 12(b) for the gluon induced width, the overall value of the width for $\Upsilon$ is smaller than that of $J / \psi$. With an upper limit of thermal mass of $600 \mathrm{MeV}$ and at temperature of $1.65 T_{c}$, the sum of the LO and NLO thermal widths is less than 50 $\mathrm{MeV}$. At this temperature, the effective dissociation cross section by a quark is less than $0.2 \mathrm{mb}$ and by a gluon than $0.6 \mathrm{mb}$. Therefore, unlike the $J / \psi$ case, the $\Upsilon$ has a smaller thermal width and effective dissociation cross section, and will effectively start accumulating at higher temperatures. Fig. 13(b) is the sum of LO and NLO 


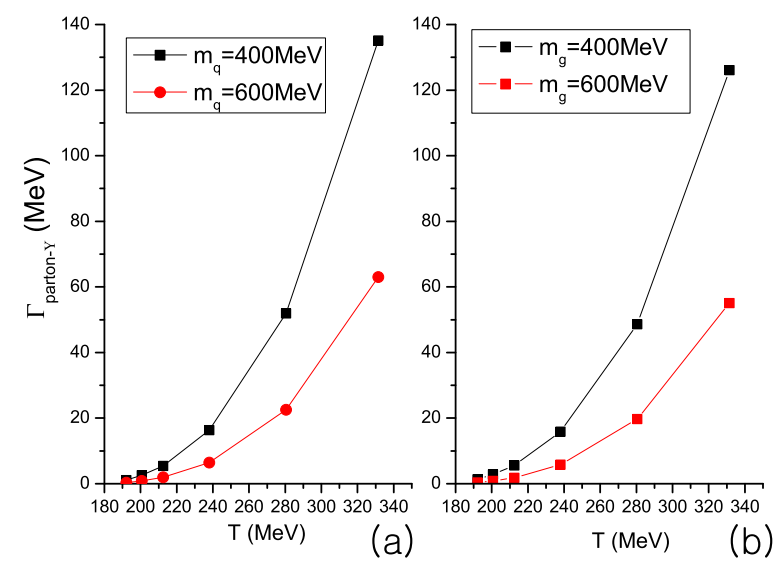

FIG. 12: (Color online) Effective thermal width of $\Upsilon$ at NLO induced (a) by quarks and (b) by gluons.

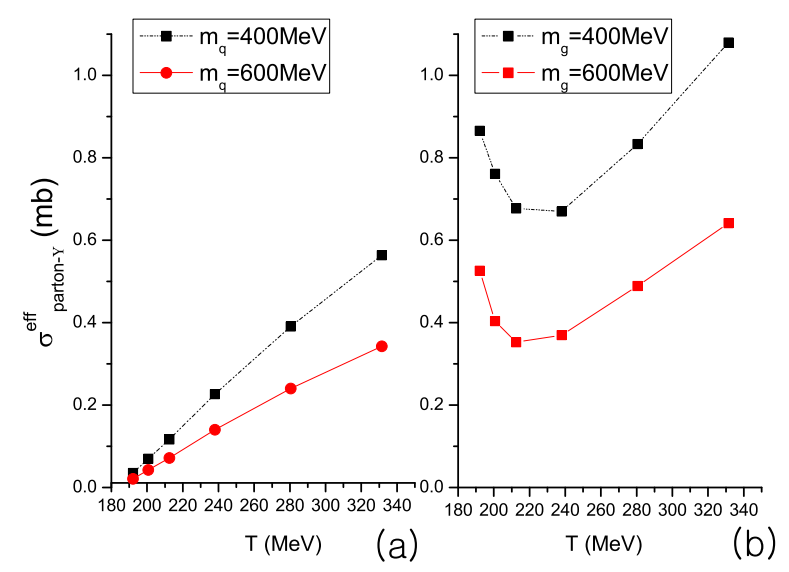

FIG. 13: (Color online) Effective cross-section of $\Upsilon$ due to (a) a quark and (b) a gluon.

effective cross section of $\Upsilon$ due to gluon. Because the contribution of LO is not negligible, the shape is quite different from the effective cross section due to quarks of Fig 13(a).

\begin{tabular}{|c|c|c|c|c|c|c|}
\hline$T / T_{c}$ & 1.13 & 1.18 & 1.25 & 1.4 & 1.65 & 1.95 \\
\hline$\epsilon_{0}(\mathrm{MeV})$ & 313 & 247 & 203 & 150 & 111 & 86 \\
\hline$\sqrt{<r^{2}>}(\mathrm{fm})$ & 0.294 & 0.331 & 0.366 & 0.425 & 0.494 & 0.562 \\
\hline$a_{0}(\mathrm{fm})$ & 0.17 & 0.19 & 0.21 & 0.246 & 0.285 & 0.324 \\
\hline
\end{tabular}

TABLE II: Binding energy and rms of the $\Upsilon$.

\section{SUMMARY}

In this work, we have calculated the thermal widths of $J / \psi$ and $\Upsilon$ at finite temperature using the elementary parton-quarkonium dissociation cross section at NLO in QCD and assuming thermal partons with effective thermal mass. We find that for $J / \psi$ at $1.4 T_{c}$, the thermal width will be 100 to $250 \mathrm{MeV}$, which translates into an effective thermal cross section of several mb. However, the corrsponding width and effective cross section for the $\Upsilon$ is much smaller. Recently Mocsy and Petreczky [24] have also calculated the thermal widths of charmonium and bottonium in QGP, assuming that the quarkonium and its constituents are in thermal equilibrium with the surrounding. The thermal width estimated by MP is similar to ours for the bottonium but several times larger for the charmonium. The result for the charmonium by MP is obtained using a phenomenological formula obtained when the binding energy is much smaller than the temperature; hence further work has to be performed to understand the discrepancy.

\section{ACKNOWLEDGEMENT}

The work was supported by Korea Research Foundation KRF-2006-C00011 and in part by the US National Science Foundation under contract number NSF-INT0327497 with the University of Tennessee. The work of SHL was also supported by the Yonsei University Research Grant. We would like to thank C. M. Ko, and R. Rapp for useful discussion.

* Electronic address: sfy@yonsei.ac.kr

$\dagger$ Electronic address: hellmare@yonsei.ac.kr

‡ Electronic address: songtsoo@yonsei.ac.kr

$\S$ Electronic address: suhoung@phya.yonsei.ac.kr

ฯ Electronic address: cyw@ornl.gov [b]

[1] T. Matsui and H. Satz, Phys. Lett. B 178, 416 (1986).

[2] A. Adare [PHENIX Collaboration], arXiv:nucl-ex/0611020.

[3] M. Gazdzicki and M. I. Gorenstein, Phys. Rev. Lett. 83, 4009 (1999) arXiv:hep-ph/9905515.

[4] P. Braun-Munzinger and J. Stachel, Phys. Lett. B 490, 196 (2000) arXiv:nucl-th/0007059.

[5] A. Andronic, P. Braun-Munzinger, K. Redlich and J. Stachel, arXiv:nucl-th/0611023.

[6] R. L. Thews, J. Phys. G 32, S401 (2006) and references cited therein.

[7] M. Asakawa, T. Hatsuda and Y. Nakahara, Prog. Part. Nucl. Phys. 46, 459 (2001) arXiv:hep-lat/0011040.

[8] M. Asakawa and T. Hatsuda, Phys. Rev. Lett. 92, 012001 (2004) arXiv:hep-lat/0308034.

[9] S. Datta, F. Karsch, P. Petreczky and I. Wetzorke, Phys. Rev. D 69, 094507 (2004) arXiv:hep-lat/0312037. 
[10] S. Datta, F. Karsch, P. Petreczky and I. Wetzorke, J. Phys. G 31, S351 (2005) arXiv:hep-lat/0412037.

[11] S. Datta, A. Jakovac, F. Karsch and P. Petreczky, AIP Conf. Proc. 842, 35 (2006) arXiv:hep-lat/0603002.

[12] Bhanot G and Peskin M E 1979 Nucl. Phys. B156 391

[13] T. H. Hansson, S. H. Lee and I. Zahed, Phys. Rev. D 37, 2672 (1988).

[14] Kharzeev D and Satz H 1994 Phys. Lett. B 334 155; Kharzeev D, Satz H, Syamtomov A and Zinovjev G 1996 Phys. Lett. B 389595.

[15] Arleo F, Gossiaux P-B, Gousset T and Aichelin J 2002 Phys. Rev. D 65014005.

[16] C. Y. Wong, Phys. Rev. C 72, 034906 (2005) arXiv:hep-ph/0408020.

[17] D. Blaschke, O. Kaczmarek, E. Laermann and V. Yudichev, Eur. Phys. J. C 43, 81 (2005)
arXiv:hep-ph/0505053.

[18] M. E. Peskin, Nucl. Phys. B 156365 (1979).

[19] L. Grandchamp and R. Rapp, Phys. Lett. B 523, 60 (2001); L. Grandchamp and R. Rapp, Nucl. Phys. A 709, 415 (2002).

[20] T. Song and S. H. Lee, Phys. Rev. D 72, 034002 (2005) arXiv:hep-ph/0501252.

[21] S. H. Lee, Y. Park, K. I. Kim and T. Song, to be published in J. Phys. Conf. Ser. arXiv:nucl-th/0701043.

[22] P. Levai and U. W. Heinz, Phys. Rev. C 57, 1879 (1998) arXiv:hep-ph/9710463.

[23] Y. S. Oh, S. Kim and S. H. Lee, Phys. Rev. C 65, 067901 (2002) arXiv:hep-ph/0111132.

[24] A. Mocsy and P. Petreczky, arXiv:0706.2183 [hep-ph]. 